plausibly predispose affected men to $\mathrm{BPH}$ associated hematuria; finasteride treatment might prevent such bleeding and could reduce morbidity and need for surgery.

Increased duration of finasteride treatment correlated positively with the decline in PSA values and with suppression of angiogenesis; these effects were evident after only 10 weeks of finasteride treatment (by contrast, clinical reductions in BPH-related hematuria require 6 months of finasteride treatment). Lekas and colleagues postulate that finasteride acts principally through hypoxia-inducible factor $1 a$ to reduce vascular endothelial growth factor expression, which results in reduced microvessel density.

Original article Lekas AG et al. (2006) Finasteride effects on hypoxia and angiogenetic markers in benign prostatic hyperplasia. Urology 68: 436-441

\section{New device that objectively measures ejaculatory latency time}

Ejaculatory latency time (ELT) is used in clinical trials to assess premature ejaculation; however, ELT is difficult to measure objectively, and there are no published normal ranges of ELT. A UK team has recently developed the Sexual Assessment Monitor, a portable device that is attached to the patient and measures the time to ejaculation after stimulation provided by the device. Three studies have recently been performed that demonstrated the device's safety and provided data on ELT in normal volunteers and in men with premature ejaculation problems.

A pooled analysis was performed on data from 53 normal, healthy volunteers and 58 men with premature ejaculation aged 18-75 years. After the results were adjusted for differences between the studies, mean ELT was significantly shorter in patients than in volunteers (131s versus $375 \mathrm{~s}, P<0.001)$. The ELT range for patients was 84-202s, and the range for volunteers was $245-574 \mathrm{~s}$. No adverse events related to use of the device were reported.

The other main diagnostic criterion used to assess premature ejaculation is intravaginal ELT, which this device is not designed to measure; however, work is underway to establish the relationship between ELT and intravaginal ELT. The authors propose that, after further validation, use of the Sexual Assessment Monitor could become the gold standard technique for diagnosis of premature ejaculation.

Original article Wyllie MG et al. (2006) Evaluation of the sexual assessment monitor, a diagnostic device used to electronically quantify ejaculatory latency time: findings from three studies. BJU Int 98: 613-618

\section{New complication associated with collagen injections for incontinence}

In a new study, Knudson et al. have identified a novel late complication that can result from endoscopic injections of collagen for pediatric urinary incontinence-calcifications of the bladder neck or urethra. Although collagen injection has lost popularity as a treatment for pediatric incontinence in recent years because of concerns about its durability, many patients underwent the procedure in the past and might be at risk of calcifications.

The authors reviewed the medical records of all 31 of their patients (average age at first injection 10 years) who had received collagen injections for urinary incontinence in 1994-1999. They found that $13 \%$ of these patients had developed submucosal calcifications of the bladder neck or proximal urethra. These patients had all received 2-3 injections, and the average total volume of collagen injected was greater for these patients than for patients without evidence of calcifications (21 $\mathrm{ml}$ versus $12 \mathrm{ml}$, respectively, $P=0.012$ ). The calcifications were diagnosed a mean of 8.8 years after the patients' first injection.

The etiology of the calcifications is unknown, as analyses were not performed to determine whether there was residual collagen in the calcifications. It is recommended that all patients with a history of endoscopic collagen injection for pediatric urinary incontinence be periodically assessed for this complication.

Original article Knudson MJ et al. (2006) Calcification of glutaraldehyde cross-linked collagen in bladder neck injections in children with incontinence: a long-term complication. J Urol 176: 1143-1146

\section{Sildenafil is safe and effective in recipients of renal transplants}

There have been few studies of sildenafil in renalallograft recipients with erectile dysfunction, and 